\title{
NON EXISTENCE OF GLOBAL SOLUTIONS OF PARABOLIC EQUATION IN CONICAL DOMAINS
}

By

\author{
Toshihiko HAMADA
}

\section{Introduction.}

Let $D$ be an unbounded domain in $\boldsymbol{R}^{N}$ and $T>0$. In this paper we study the initial-boundary value problem

$$
\begin{array}{ll}
u_{t}=\Delta u+|x|^{\sigma} u^{p} & \text { in } D \times(0, T), \\
u(x, t)=0 & \text { on } \partial D \times(0, T), \\
u(x, 0)=u_{0}(x) & \text { in } D,
\end{array}
$$

where $\sigma \geqq 0, p>1, u_{0} \geqq 0,\langle x\rangle^{\sigma /(p-1)} u_{0}\left(\langle x\rangle=\sqrt{1+|x|^{2}}\right)$ is continuous and bounded in $\bar{D}$ and $u_{0}=0$ on $\partial D$.

When $D=\boldsymbol{R}^{N}$ and $\sigma=0$, Fujita [1] and Weissler [2] proved that if $1<p \leqq$ $1+2 / N$, there is no nontrivial nonnegative global solution of $(\mathrm{P})$.

When $D$ is a cone with vertex at the origin, that is $D=\left\{x \in \boldsymbol{R}^{N} \backslash 0 ; x /|x|\right.$ $\in \Omega\}$, where $\Omega \subset S^{N-1}$ is an open connected subset with smooth boundary, Levine and Meier, [3] and [4], proved that if $1<p<1+(2+\sigma) /\left(N+\gamma_{+}\right)$and $\sigma \geqq 0$, or $p=1+2 /\left(N+\gamma_{+}\right)$and $\sigma=0$, there is no nontrivial nonnegative global solution of $(\mathrm{P})$, where $\gamma_{+}$is the positive root of $\gamma(\gamma+N-2)=\omega_{1}$, and $\omega_{1}$ is the smallest Dirichlet eigenvalue for the Laplace-Beltrami operator on $\Omega$.

In this paper we shall prove that there is no nontrivial global solution of (P) if $\sigma>0$ and $p=1+(2+\sigma) /\left(N+\gamma_{+}\right)$are valid. Moreover we can prove that when $D=\boldsymbol{R}^{N}$ there is no nontrivial global solution if $1+\sigma /(N-2) \leqq p \leqq 1+$ $(2+\sigma) / N$ and $\sigma>0$.

Definition 1.1. For $T>0, u=u(x, t)$ is called a solution of $(\mathrm{P})$ in $(0, T)$, if

(A) $u$ is continuous in $\bar{D} \times[0, T)$,

(B) $u_{t}, u_{x_{i}}$ and $u_{x_{i} x_{j}}(i, j=1, \cdots, N)$ are continuous in $D \times(0, T)$,

(C) $\|u(t)\|_{\sigma /(p-1)}$ is finite for each $t \in[0, T)$,

(D) $u$ satisfies $(\mathrm{P})$,

where $\|u(t)\|_{\sigma /(p-1)}:=\sup _{D}\langle x\rangle^{\sigma /(p-1)}|u(u, t)|$.

Received February 18, 1993. Revised June 7, 1992. 
Similarly, $\underline{u}$ is called a subsolution of $(\mathrm{P})$ in $(0, T)$, if $\underline{u}$ satisfies (A), (B), (C) and

$(\underline{P})$

$$
\begin{array}{ll}
\underline{u}_{t} \leqq \Delta \underline{u}+|x|^{\sigma} \underline{\underline{u}}^{p} & \text { in } D \times(0, T), \\
\underline{u}(x, t)=0 & \text { on } \partial D \times(0, T), \\
\underline{u}(x, 0)=u_{0}(x) & \text { in } D .
\end{array}
$$

Definition 1.2. $\bar{T}:=\sup \left\{T>0 ;\|u(t)\|_{\sigma /(p-1)}\right.$ is finite for $\left.0 \leqq t<T\right\}$ is called the nontrivial existence time of $u$. If $\bar{T}=+\infty$, then $u$ is called a global solution of $(\mathrm{P})$.

REMARK. If $0<T<\infty$ and $\|u(t)\|_{\sigma /(p-1)}$ is finite on $(0, T)$, the solution $u$ can be extended beyond $T$ (see Theorem 1.1).

We begin with stating the local existence theorem for $(P)$.

THEOREM 1.1. Let $D$ be a cone in $\boldsymbol{R}^{N}$. Then for any nonnegative function $u_{0}$ in $C^{0}(\bar{D})$ satisfying $\left\|u_{0}\right\|_{\sigma /(p-1)}<\infty$ and $u_{0}=0$ on $\partial D$ there is a solution $u(x, t)$ of $(\mathrm{P})$ in $\left(0, t_{0}\right)$ such that $\|u(t)\|_{\sigma /(p-1)}$ is finite in $\left(0, t_{0}\right)$ where $t_{0}>0$ depends only on $\sigma, p, N$ and $\left\|u_{0}\right\|_{\sigma /(p-1)}$.

The main two theorem in this paper are the following.

THEOREM 1.2. Let $D$ be a cone in $\boldsymbol{R}^{N}, N \geqq 3$. If $u_{0} \geqq 0$ and $u_{0} \neq \equiv, p=1+$ $(2+\sigma) /\left(N+\gamma_{+}\right)$and $0<\sigma \leqq 2(N-2) /\left(\gamma_{+}+2\right)$, there is no global solution.

THEOREM 1.3. Let $D=\boldsymbol{R}^{N}, N \geqq 3$. If $u_{0} \geqq 0$ and $u_{0} \neq 0,1+\sigma /(N-2) \leqq p \leqq$ $1+(2+\sigma) / N$ and $0<\sigma \leqq N-2$, there is no global solution.

To prove Theorem 1.2 and Theorem 1.3, we need the following estimate

$$
v(x, t)<\left((p-1)|x|^{\sigma} t\right)^{-1 /(p-1)} \quad \text { for } 0<t<T,
$$

where $T>0$ is the maximum existence time of the solution of $(\mathrm{P})$ and $v(x, t)$ is the solution of the heat equation with the same initial and boundary condition as $(\mathrm{P})$.

The above inequality is true provided $0<\sigma /(p-1) \leqq N-2$ (see Lemma 3.2).

\section{Proof of Theorem 1.1.}

Throughout this paper we take advantage of the following proposition proved by Protter and Weinberger [5] (Theorem 10, p.p. 183-184). 
Proposition 2.1. Let $D$ be an unbounded domain in $\boldsymbol{R}^{N}$. If $u_{t}$ and $u_{x_{i} x_{j}}$ $(i, j=1, \cdots, N)$ are exist and continuous in $D \times(0, T)$ and $u=u(x, t)$ satisfies the following inequalities

$$
\begin{array}{ll}
u_{t} \leqq \Delta u+h u & \text { in } D \times(0, T), \\
u(x, t) \leqq 0 & \text { on } \partial D \times(0, T), \\
u(x, 0) \leqq 0 & \text { in } D,
\end{array}
$$

where $h=h(x, t)$ is bounded in $D \times[0, T)$. If there exist $c>0$ such that $\underline{\lim }_{r \rightarrow \infty} e^{-c r^{2}}$ - $\left\{\max _{|x|=r, 0 \leq t \leq T} u(x, t)\right\} \leqq 0$, then $u(x, t) \leqq 0$ in $D \times(0, T)$.

REMARK. In the case $D=\boldsymbol{R}^{N}$, we can eliminate the boundary condition of $u$.

We introduce the Green's function $G(x, y ; t)=G(r, \theta, \rho, \phi ; t)(r=|x|, \rho=|y|$, $\theta=x /|x| \in \Omega, \phi=y /|y| \in \Omega)$, for the linear heat equation in the cone.

Let $\left\{\psi_{n}(\theta)\right\}_{n=1}^{\infty}$ be a normalized orthogonal system for $\Delta_{\theta}$ on $\Omega$ corresponding to the sequence $\left\{\omega_{n}\right\}$ of Dirichlet eigenvalues for this problem, especially we take $\psi_{1}>0$ in $\Omega$ and $\int_{\Omega} \psi_{1}(\theta) d S_{\theta}=c_{1}$.

Here

$$
\begin{aligned}
G(x, y, t) & =G(r, \theta, \rho, \phi ; t) \\
& =\frac{1}{2 t}(r \rho)^{-(N-2) / 2} \exp \left(-\frac{\rho^{2}+r^{2}}{4 t}\right) \sum_{n=1}^{\infty} I_{\nu_{n}}\left(\frac{r \rho}{2 t}\right) \psi_{n}(\theta) \psi_{n}(\phi),
\end{aligned}
$$

where $\nu_{n}=\left\{((N-2) / 2)^{2}+\omega_{n}\right\}^{1 / 2}$, and

$$
I_{\nu}(z)=\left(\frac{z}{2}\right)^{\nu} \sum_{k=0}^{\infty} \frac{(z / 2)^{2 k}}{k ! \Gamma(\nu+k+1)} \sim \begin{cases}(z / 2)^{\nu} / \Gamma(\nu+1) & z \rightarrow 0^{+} \\ e^{z} / \sqrt{2 \pi z} & z \rightarrow+\infty\end{cases}
$$

(see Watson [6]).

Lemma 2.2. Let $D$ be a cone in $\boldsymbol{R}^{N}$. Assume that $v_{0}$ is a bounded continuous function in $D$ and vanishes on $\partial D$. Then there exist a unique solution of the heat equation

$$
\begin{array}{ll}
v_{t}=\Delta v & \text { in } D \times(0, \infty), \\
v(x, t)=0 & \text { on } \partial D \times(0, \infty), \\
v(x, 0)=v_{0}(x) & \text { in } D,
\end{array}
$$

in $C(\bar{D} \times[0, \infty)) \cap C^{2}(D \times(0, \infty))$, which has the form

$$
v(x, t)=\int_{D} G(x, y ; t) v_{0}(y) d y .
$$

Especially, if $v_{0}(x) \geqq 0$ in $D$, then $v(x, t) \geqq 0$ in $D \times(0, \infty)$. 
LEMMA 2.3. Let $v=v(x, t)$ be a solution of (2.2) and $\alpha:=\max \{0,-(\sigma / 2(p-1))$ $\cdot(N-2-\sigma /(p-1))\}$. If $\|v\|_{\sigma /(p-1)}<\infty$, then for $0<t<\infty$

$$
\|v(t)\|_{\sigma /(p-1)} \leqq\left\|v_{0}\right\|_{\sigma /(p-1)} \exp (\alpha t) .
$$

REMARK. Moreover if we take $0<t_{0} \leqq(\log 2) / \alpha$, then for $0<t<t_{0}$

$$
\|v(t)\|_{\sigma /(p-1)} \leqq 2\left\|v_{0}\right\|_{\sigma /(p-1)} .
$$

ProOF OF LemMA 2.3. Let $w(x, t):=v(x, t)-\left\|v_{0}\right\|_{\sigma /(p-1)}\langle x\rangle^{-\sigma /(p-1)} \exp (\alpha t)$, then we have

$$
\begin{aligned}
\Delta w-w_{t} & =\left\{\alpha|x|^{4}+\{2 \alpha\right. \\
& \left.\left.+\frac{\sigma}{p-1}\left(N-2-\frac{\sigma}{p-1}\right)\right\}|x|^{2}+\frac{N \alpha}{p-1}+\alpha\right\} \\
& \quad \times 0 .
\end{aligned}
$$

Combining this with Proposition 2.1, we get $w(x, t) \leqq 0$ for $D \times[0, \infty)$. This shows Lemma 2.3.

Proof of Theorem 1.1. Define $\alpha:=\max \{0,-(\sigma / 2(p-1))(N-2-\sigma /(p-1))\}$ and $t_{0}:=\min \left\{(\log 2) / \alpha, 4^{p}\left(\left\|u_{0}\right\|_{\sigma /(p-1)}\right)^{1-p}\right\}$.

First, we consider the following initial-boundary value problem

$$
\begin{array}{ll}
\partial_{t} V_{1}=\Delta V_{1}+|x|^{\sigma} V_{0}^{p} & \text { in } D \times\left(0, t_{0}\right), \\
V_{1}(x, t)=0 & \text { on } \partial D \times\left(0, t_{0}\right), \\
V_{1}(x, 0)=u_{0}(x) & \text { in } D,
\end{array}
$$

where $V_{0}$ is a solution of (2.2) with the initial condition $v_{0}=u_{0}$.

The solution of $\left(\mathrm{P}_{1}\right)$ is

$$
V_{1}(x, t)=V_{0}(x, t)+\int_{0}^{t} \int_{D} G(x, y ; t-\tau)|y|^{\sigma} V_{0}^{p}(y, \tau) d y d \tau
$$

for $(x, t) \in D \times\left(0, t_{0}\right)$.

Since $V(x, t ; \tau):=\int_{D} G(x, y ; t-\tau)|y|^{\sigma} V_{0}^{p}(y, \tau) d y$ is a solution of (2.2) with the initial condition $v_{0}=|x|^{\sigma} V_{0}^{p}(x, \tau)$ for arbitrarily fixed $\tau \in(0, t)$, so we have $\|V(t ; \tau)\|_{\sigma /(p-1)} \leqq 2\left(2\left\|u_{0}\right\|_{\sigma /(p-1)}\right)^{p}$ for $0<\tau<t_{0}$ by using the above remark.

The solution $V_{1}$ of $\left(\mathrm{P}_{1}\right)$ satisfies 


$$
\begin{aligned}
\left\|V_{1}(t)\right\|_{\sigma /(p-1)} & \leqq\left\|V_{0}(t)\right\|_{\sigma /(p-1)}+\int_{0}^{t}\|V(t ; \tau)\|_{\sigma /(p-1)} d \tau \\
& \leqq 2\left\|u_{0}\right\|_{\sigma /(p-1)}+\int_{0}^{t} 2\left(2\left\|u_{0}\right\|_{\sigma /(p-1)}\right)^{p} d \tau \\
& \leqq 4\left\|u_{0}\right\|_{\sigma /(p-1)} \quad \text { for } 0<t<t_{0} .
\end{aligned}
$$

Next we consider the following problem

$$
\begin{array}{lll} 
& \partial_{t} V_{i+1}(x, t)=\Delta V_{i+1}(x, t)+|x|^{\sigma} V_{i}^{p}(x, t) & \text { in } D \times\left(0, t_{0}\right), \\
\left(\mathrm{P}_{i+1}\right) & V_{i+1}(x, t)=0 & \text { on } \partial D \times\left(0, t_{0}\right), \\
& V_{i+1}(x, 0)=u_{0}(x) & \text { in } D,
\end{array}
$$

where $i=1,2, \cdots$.

Then

$$
\left\|V_{i}(t)\right\|_{\sigma /(p-1)} \leqq 4\left\|u_{0}\right\|_{\sigma /(p-1)} \quad \text { for } 0<t<t_{0} .
$$

As can be seen from the argument to obtain the estimate of $V_{1}$, the above inequality is true for $V_{i+1}$.

Moreover we can obtain

$$
\left\|V_{i+1}(t)-V_{i}(t)\right\|_{\sigma /(p-1)} \leqq \int_{0}^{t} 2 p\left(4\left\|u_{0}\right\|_{\sigma /(p-1)}\right)^{p-1} \mathscr{M}_{i}(\tau) d \tau,
$$

where $\mathscr{M}_{i}(\tau):=\sup _{0<s<r}\left\|V_{i}(s)-V_{i-1}(s)\right\|_{\sigma /(p-1)}(i=1,2, \cdots)$, because

$$
\begin{aligned}
& \langle x\rangle^{\sigma /(p-1)} \|\left. x\right|^{\sigma} V_{i}^{p}(x, \tau)-|x|^{\sigma} V_{i-1}^{p}(x, \tau) \mid \\
& \quad \leqq 2 p\left(4\left\|u_{0}\right\|_{J /(p-1)}\right)^{p-1}\langle x\rangle^{\sigma /(p-1)}\left|V_{i}(x, \tau)-V_{i-1}(x, \tau)\right| \\
& \quad \leqq 2 p\left(4\left\|u_{0}\right\|_{\sigma /(p-1)}\right)^{p-1} \mathscr{M}_{i}(\tau) .
\end{aligned}
$$

So,

$$
\mathscr{M}_{i+1}(t) \leqq \int_{0}^{t} 2 p\left(4\left\|u_{0}\right\|_{\sigma /(p-1)}\right)^{p-1} \mathscr{M}_{i}(\tau) d \tau \quad i=1,2, \cdots
$$

Note that $\mathscr{M}_{1}(t) \leqq 2\left(2\left\|u_{0}\right\|_{\sigma /(p-1)}\right)^{p} t$.

Thus,

$$
\mathscr{M}_{i}(t) \leqq \frac{2^{2-p}\|u(t)\|_{\sigma /(p-1)}\left(2 p\left(2\left\|u_{0}\right\|_{\sigma /(p-1)}\right)^{p-1} t\right)^{i}}{i ! p} \quad i=1,2, \cdots .
$$

We conclude that there is a solution $u$ of $(\mathrm{P})$ such that $\left\{V_{i}\right\}$ converges to $u$ uniformly in $D \times\left(0, t_{0}\right)$. It is clear that $u$ is the unique solution of $(\mathrm{P})$.

\section{Proof of Theorem 1.2 .}

LEMMA 3.1. Let $T>0$ be the existence time of $u$, and $\underline{u}$ a subsolution in $\left(0, T_{1}\right)$ for some $T_{1}>0$. Then we have 


$$
\underline{u}(x, t) \leqq u(x, t) \quad \text { in } D \times\left[0, T_{2}\right),
$$

where $T_{2}=\min \left\{T_{1}, T\right\}$.

PrOOF. Let

$$
U(x, t):=\underline{u}(x, t)-u(x, t),
$$

then $U_{t} \leqq \Delta U+|x|^{\sigma}\left(\underline{u}^{p}-u^{p}\right)$.

By using the mean value theorem, there exist $0<\zeta<1$ such that

$$
|x|^{\circ}\left(\underline{u}^{p}-u^{p}\right)=h(x, t)(\underline{u}-u)
$$

where,

$$
\begin{aligned}
h(x, t) & =p|x|^{\sigma}\{(1-\zeta) \underline{u}+\zeta u\}^{p-1} \\
& \leqq p \max \left\{\|\underline{u}(t)\|_{\sigma /(p-1)},\|u(t)\|_{\sigma /(p-1)}\right\}^{p-1}
\end{aligned}
$$

Combining this and Proposition 2.1, we get

$$
U(x, t) \leqq 0 \quad \text { in } D \times\left[0, T_{2}\right) .
$$

LEMMA 3.2. We assume $0<\sigma /(p-1) \leqq N-2$. Let $\bar{T}$ be the maximal existence time of $u$, and $v$ be the solution of the linear heat equation with the same initialboundary condition as $u$.

Then

$$
v(x, t)<\left((p-1)|x|^{\sigma} t\right)^{-1 /(p-1)} \quad \text { in } D \times(0, \bar{T}) .
$$

PROOF. If $\bar{T} \leqq T_{0}:=\left(\left\|u_{0}\right\|_{\sigma /(p-1)}^{(p-1)}\right) /(p-1)$, then by Lemma 2.3

$$
\begin{aligned}
v(x, t) & \leqq\left\|u_{0}\right\|_{\sigma /(p-1)}\langle x\rangle^{-\sigma /(p-1)} \\
& \leqq((p-1) \bar{T})^{-1 /(p-1)}\langle x\rangle^{-\sigma /(p-1)} \\
& <\left((p-1)|x|^{\sigma} t\right)^{-1 /(p-1)} \quad \text { for } 0 \leqq t<\bar{T} .
\end{aligned}
$$

Now we assume $\bar{T}>T_{0}$ and let

$$
\underline{u}(x, t)=\left\{(v(x, t))^{-(p-1)}-(p-1)|x|^{\sigma} t\right\}^{-1 /(p-1)} .
$$

We shall prove $\|\underline{u}(t)\|_{\sigma /(p-1)}$ is finite for $0<t<\bar{T}$.

When $0<t<T_{0}$, from Lemma 2.3, we see that

$$
\|\underline{u}(t)\|_{\sigma /(p-1)} \leqq\left\{\left\|u_{0}\right\|_{\sigma /(p-1)}-(p-1) t\right\}^{-1 /(p-1)}<\infty .
$$

We assume that there exists $\tau<\bar{T}$ such that $\|\underline{u}\|_{\sigma /(p-1)} \rightarrow \infty$ as $t \uparrow \tau$, and let $t_{1}$ be the smallest one of all such $\tau$.

On the other hand, let $t_{2}$ be an arbitrary constant with $0<t_{2}<t_{1}$. Then $\underline{u}$ satisfies for $t \leqq t_{2}$, 


$$
\begin{aligned}
\Delta \underline{u} & +|x|^{\sigma} \underline{u}^{p}-\underline{u}_{t} \\
= & \sigma(N-2-\sigma /(p-1))|x|^{\sigma-2} t\left\{v^{-(p-1)}-(p-1)|x|^{\sigma} t\right\}^{-p /(p-1)} \\
& +p(p-1) v^{-(p+1)}|x|^{\sigma} t \sum_{i=1}^{N}\left(v_{i}+\frac{\sigma}{p-1} \frac{x_{i}}{|x|^{2}} v\right)^{2}\left\{v^{-(p-1)}-(p-1)|x|^{\sigma} t\right\}^{(-2 p+1) /(p-1)} \\
\geqq 0 . &
\end{aligned}
$$

So $\underline{u}$ is a subsolution for $0<t<t_{2}<t_{1}$, it follows from Lemma 3.1 that $\underline{u}(x, t) \leqq$ $u(x, t)$ in $D \times\left[0, t_{2}\right)$. Hence we see from the definition of $\|u(t)\|_{\sigma /(p-1)}$,

$$
\|\underline{u}(t)\|_{\sigma /(p-1)} \leqq\|u(t)\|_{\sigma /(p-1)} \quad \text { for } 0<t \leqq t_{2} .
$$

On the other hand,

$$
\left\|\underline{u}\left(t_{2}\right)\right\|_{\sigma /(p-1)} \longrightarrow \infty \quad \text { as } t_{2} \uparrow t_{1} .
$$

We have reached the contradiction.

Proof of TheOREM 1.2. We assume that there exists a global solution of $(\mathrm{P})$, then from Lemma 3.2 we have

$$
(p-1)^{-1 /(p-1)}>|x|^{\sigma /(p-1)} t^{1 /(p-1)} v(x, t) \quad \text { in } D \times(0, \infty) .
$$

Integrating the above both sides over $\Omega$ with respect to $\psi_{1}(\theta) d S_{\theta}$, we can estimate by use of (2.3),

$$
\begin{aligned}
c_{2}> & \int_{\Omega} r^{\sigma /(p-1)} t^{1 /(p-1)} v(r, \theta ; t) \psi_{1}(\theta) d S_{\theta} \\
= & \int_{\Omega} r^{\sigma /(p-1)} t^{1 /(p-1)} \int_{0}^{\infty} \int_{\Omega} G(r, \theta, \rho, \phi ; t) u_{0}(\rho, \phi) \rho^{N-1} d S_{\phi} d \rho \psi_{1}(\theta) d S_{\theta} \\
= & c_{1} r^{\sigma /(p-1)} t^{1 /(p-1)} \int_{0}^{\infty} \int_{\Omega} \frac{1}{2 t}(r \rho)^{-(N-2) / 2} I_{\nu_{1}}\left(\frac{r \rho}{2 t}\right) \exp \left(-\frac{r^{2}+\rho^{2}}{4 t}\right) u_{0}(\rho, \phi) \psi_{1}(\phi) \\
& \times \int_{\Omega} \psi_{1}^{2}(\theta) \rho^{N-1} d S_{\theta} d S_{\phi} d \rho \\
\geqq & c_{3} r^{\sigma /(p-1)-(N-2) / 2} t^{1 /(p-1)-1} \exp \left(-\frac{r^{2}}{4 t}\right) \\
& \times \int_{0}^{\infty} \int_{\Omega} \rho^{-(N-2) / 2+N-1}\left(\frac{r \rho}{2 t}\right)^{\nu_{1}} \exp \left(-\frac{\rho^{2}}{4 t}\right) u_{0}(\rho, \phi) \psi_{1}(\phi) d S_{\phi} d \rho,
\end{aligned}
$$

where $c_{1}, c_{2}$ and $c_{3}$ are constants and $r=|x|, \theta=x /|x|$.

Let $r=t^{1 / 2}$. Then $\nu_{1}=\gamma_{+}+(N-2) / 2$, there exists $c_{4}>0$ independent of $t$ such that

$$
\begin{aligned}
c_{4} & >t^{\left(\sigma /(p-1)-(N-2) / 2-\nu_{1}\right) / 2+1 /(p-1)-1} \int_{0}^{\infty} \int_{\Omega} \rho^{\gamma++N-1} \exp \left(-\frac{\rho^{2}}{4 t}\right) u_{0}(\rho, \phi) \psi_{1}(\phi) d S_{\phi} d \rho, \\
& =\int_{0}^{\infty} \int_{\Omega} \rho^{\gamma_{+}+N-1} \exp \left(-\frac{\rho^{2}}{4 t}\right) u_{0}(\rho, \phi) \psi_{1}(\theta) d S_{\phi} d \rho .
\end{aligned}
$$


Since $u$ is a global solution, we can replace $u_{0}(\rho, \phi)$ by $u\left(r, \theta ; t_{0}\right)$ for any $t_{0}>0$. Thus, for $t>t_{0}$

$$
c_{4}>\int_{0}^{\infty} \int_{\Omega} r^{r_{+}+N-1} \exp \left(-\frac{r^{2}}{4\left(t-t_{0}\right)}\right) u\left(r, \theta ; t_{0}\right) \psi_{1}(\theta) d S_{\theta} d r .
$$

Here let $t \uparrow \infty$ and replace $t_{0}$ by $t$. Then there exists $c_{5}>0$ such that

$$
\begin{aligned}
c_{4} \geqq & \int_{0}^{\infty} \int_{\Omega} r^{r_{+}+N-1} \int_{0}^{t} \int_{0}^{\infty} \int_{\Omega} G(r, \theta, \rho, \phi ; t-s) \\
& \times \rho^{\sigma+N-1} u^{p}(r, \theta, s) d \rho d S_{\phi} d s \varphi_{1}(\theta) d S_{\theta} d r \\
\geqq & c_{5} \int_{0}^{t} \int_{0}^{\infty} \int_{\Omega} u^{p}(\rho, \phi, s) \psi_{1}(\phi)(t-s)^{-\left(\gamma_{+}+N / 2\right)} \rho^{r_{+}+\sigma+N-1} \\
& \times \exp \left(-\frac{\rho^{2}}{4(t-s)}\right) \int_{0}^{\infty} r^{2 \gamma_{+}+N-1} \exp \left(-\frac{r^{2}}{4(t-s)}\right) d r d S_{\phi} d \rho d s .
\end{aligned}
$$

From Hölder's inequality, it follows that there exists $c_{6}>0$ such that

$$
c_{6} \geqq \int_{0}^{t} \int_{0}^{\infty}\left(\int_{\Omega} u(\rho, \phi, s) \psi_{1}(\phi) d S_{\phi}\right)^{p} \rho^{\gamma_{+}+\sigma+N-1} \exp \left(-\frac{\rho^{2}}{4(t-s)}\right) d \rho d s .
$$

Moreover since we see $u(x, t) \geqq v(x, t)$ from Lemma 3.1 , we can estimate

$$
\begin{aligned}
\int_{\Omega} u(\rho, \phi, s) \phi_{1}(\phi) d S_{\phi} \\
\geqq \int_{\Omega} v(\rho, \phi, s) \phi_{1}(\phi) d S_{\phi} \\
\geqq c_{3} s^{-\left(\gamma_{+}+N / 2\right)} \rho^{\gamma_{+}} \exp \left(-\frac{\rho^{2}}{4 s}\right) \\
\quad \times \int_{0}^{\infty} \int_{\Omega} r^{r_{+}+N-1} \exp \left(-\frac{r^{2}}{4 s}\right) u_{0}(r, \theta) \phi_{1}(\theta) d S_{\theta} d r \\
\geqq c_{7} s^{-\left(r_{+}+N / 2\right)} \rho^{r_{+}} \exp \left(-\frac{\rho^{2}}{4 s}\right)
\end{aligned}
$$

for $0<t^{\prime} \leqq s$.

Thus we obtain

$$
c_{6} \geqq\left(c_{7}\right)^{p} \int_{t^{\prime}}^{t} s^{-p\left(\gamma_{+}+N / 2\right)} \int_{0}^{\infty} \rho^{(p+1) \gamma_{+}+\sigma+N-1} \exp \left(-\frac{\rho^{2}}{4 s}\left(p+\frac{s}{t-s}\right)\right) d \rho d s .
$$

Since $p>1$, we can see that for $\delta \in(0,1)$ such that $p-1+1 / \delta>0$. Noting that $p+s /(t-s)=p+t /(t-s)-1 \leqq p+1 / \delta-1$, for $s \in\left[t^{\prime},(1-\delta) t\right]$, we have $c_{8}>0$, such that 


$$
\begin{aligned}
c_{8} & \geqq \int_{t^{\prime}}^{(1-\delta) t} s^{\left((p+1) \gamma_{+}+\sigma+N\right) / 2-p\left(\gamma_{+}+N / 2\right)} d s \\
& =\int_{t^{\prime}}^{(1-\delta) t} \frac{1}{s} d s \longrightarrow \infty
\end{aligned}
$$

as $t \rightarrow \infty$.

This is a contradiction. Thus we have proved Theorem 1.2.

\section{Proof of Theorem 1.3 .}

In this section we consider next problem

$$
\begin{array}{ll}
u_{t}=\Delta u+|x|^{\sigma} u^{p} & \text { in } \boldsymbol{R}^{N} \times(0, T), \\
u(x, 0)=u_{0}(x) & \text { in } \boldsymbol{R}^{N},
\end{array}
$$

where $N \geqq 3$ and $\|u(t)\|_{\sigma /(p-1)}$ is finite and not zero.

REMARK 4.1. If $D=\boldsymbol{R}^{N}$, the statements of Lemma 3.1 and Lemma 3.2 are also true without the boundary condition of $(\mathrm{P})$.

Proof of TheOREM 1.3. We assume that there exists a global solution $u=u(x, t)$ of (4.1) such that $\|u(t)\|_{\sigma /(p-1)}$ is finite for any $t>0$. Moreover, let $v=v(x, t)$ be a solution of the heat equation with an initial datum $u_{0}(x)$. Since $|x-y|^{2} \leqq 2\left(|x|^{2}+|y|^{2}\right)$ we have

$$
\begin{aligned}
v(x, t) & =\int_{R^{N}}\left(\frac{1}{2 \sqrt{\pi t}}\right)^{N} \exp \left(-\frac{|x-y|^{2}}{4 t}\right) u_{0}(y) d y \\
& \geqq \int_{R^{N}}\left(\frac{1}{2 \sqrt{\pi t}}\right)^{N} \exp \left(-\frac{|x|^{2}+|y|^{2}}{2 t}\right) u_{0}(y) d y .
\end{aligned}
$$

By use of Lemma 3.2 we have

$$
(p-1)^{-1 /(p-1)}|x|^{-\sigma /(p-1)} t^{-1 /(p-1)} \geqq\left(\frac{1}{2 \sqrt{\pi t}}\right)^{N} \exp \left(-\frac{|x|^{2}}{2 t}\right) \int_{R^{N}} \exp \left(-\frac{|y|^{2}}{2 t}\right) u_{0}(y) d y .
$$

Therefore, for $|x|=t^{1 / 2}$ we have

$$
2^{N}(p-1)^{-1 /(p-1)} \pi^{N / 2} \exp \left(\frac{1}{2}\right) t^{(N-(2+\sigma) /(p-1)) / 2} \geqq \int_{R^{N}} \exp \left(-\frac{|y|^{2}}{2 t}\right) u_{0}(y) d y .
$$

If $p<1+(2+\sigma) / N$, the left side of the above inequality goes to 0 as $t \rightarrow \infty$. This is a contradiction.

Next, we assume $p=1+(2+\sigma) / N$ and let $c_{9}=2^{N}(p-1)^{-1 /(p-1)} \pi^{N / 2} \exp (1 / 2)$. Then

$$
c_{9} \geqq \int_{R^{N}} \exp \left(-\frac{|y|^{2}}{2 t}\right) u_{0}(y) d y \text {. }
$$


As we discussed in the proof of Theorem 1.2 we can replace $u_{0}(y)$ by $u\left(y, t_{0}\right)$ for arbitrarily $t_{0}>0$. Thus,

$$
c_{9} \geqq \int_{R^{N}} \exp \left(-\frac{|y|^{2}}{2\left(t-t_{0}\right)}\right) u\left(y, t_{0}\right) d y \quad \text { for } t>t_{0} .
$$

The right side of the above inequality goes to

$$
\int_{R^{N}} u\left(y, t_{0}\right) d y \quad \text { as } t \rightarrow \infty .
$$

Replace $t_{0}$ by $t$. Then we have

$$
\begin{aligned}
c_{9} & \geqq \int_{R^{N}} u(y, t) d y \\
& \geqq \int_{R^{N}} \int_{0}^{t} \int_{R^{N}}\left(\frac{1}{2 \sqrt{\pi(t-s)}}\right)^{N} \exp \left(-\frac{|x-y|^{2}}{4(t-s)}\right)|y|^{p} u^{p}(y, s) d y d s d x \\
& \geqq \int_{0}^{t} \int_{R^{N}}|y|^{\sigma} u^{p}(y, s) \int_{R^{N}}\left(\frac{1}{2 \sqrt{\pi(t-s)}}\right)^{N} \exp \left(-\frac{|x-y|^{2}}{4(t-s)}\right) d x d y d s \\
& =\int_{0}^{t} \int_{R^{N}}|y|^{\sigma} u^{p}(y, s) d y d s \\
& =\int_{0}^{t} \int_{0}^{\infty} \int_{S^{N-1}} u^{p}(\rho, \phi, s) \rho^{\sigma+N-1} d S_{\phi} d \rho d s .
\end{aligned}
$$

By Hölder's inequality we have $c_{10}$ such that,

$$
c_{10}>\int_{0}^{t} \int_{0}^{\infty}\left(\int_{S^{N-1}} u(\rho, \phi, s) d S_{\phi}\right)^{p} \rho^{\sigma+N-1} d \rho d s .
$$

Moreover, by using Lemma 3.2 and (4.2) we get

$$
\begin{aligned}
& \int_{S^{N-1}} u(\rho, \phi, s) d S_{\phi} \\
& \quad \geqq 2^{-N} \pi^{-N / 2} \int_{S^{N-1}} s^{-N / 2} \exp \left(-\frac{\rho^{2}}{2 s}\right) \int_{R^{N}} \exp \left(-\frac{|x|^{2}}{2 s}\right) u_{0}(x) d x d S_{\phi} .
\end{aligned}
$$

Since $u_{0} \not \equiv 0$, for any $t^{\prime}>0$ there exists $c_{11}=c_{11}\left(u_{0} ; t^{\prime}\right)>0$ such that

$$
c_{11}<2^{-N} \pi^{-N / 2} \int_{S^{N-1}} \int_{R^{N}} \exp \left(-\frac{|x|^{2}}{2 s}\right) u_{0}(x) d x d S_{\phi} \quad \text { for } s \geqq t^{\prime} .
$$

Hence we get

$$
\begin{aligned}
c_{10} & >\int_{t^{\prime}}^{t} \int_{0}^{\infty}\left(c_{11} s^{-N / 2} \exp \left(-\frac{\rho^{2}}{2 s}\right)\right)^{p} \rho^{\sigma+N-1} d \rho d s \\
& =\left(c_{11}\right)^{p} \int_{t^{\prime}}^{t} \int_{0}^{\infty} \rho^{\sigma+N-1} \exp \left(-\frac{p \rho^{2}}{2 s}\right) d \rho s^{-(N p) / 2} d s \\
& =\left(c_{11}\right)^{p} \frac{1}{2}\left(\frac{2}{p}\right)^{(\sigma+N) / 2} \Gamma\left(\frac{\sigma+N}{2}\right) \int_{t^{\prime}}^{t} s^{(\sigma+N) / 2-(N p) / 2} d s .
\end{aligned}
$$


Since $p=1+N /(\sigma+2)$, the right side of the above inequality goes to $\infty$ as $t \rightarrow \infty$. This is a contradiction. Thus we have proved Theorem 1.3.

Acknowledgement. The author woold like to acknowledge several helpful discussions with Professor K. Mochizuki and Professor K. Kajitani. The author would also like to thank Professor H. A. Levine for stimulating conversations.

\section{References}

[1] H. Fujita, On the blowing up of solutions of the Cauchy problem for $u_{t}=\Delta u+u^{1+\alpha}$, J. Fac. Sci. Univ. Tokyo Sect. IV Math. 13 (1966), 109-124.

[2] F.B. Weissler, Existence and nonexistence of global solutions for a semilinear heat equation, Israel J. Math. 38 (1981), 29-40.

[3] H. A. Levine and P. Meier, The value of critical exponent for reaction-diffusion equation in cones, Arch. Ratl. Mech. Anal. 109 (1990), 73-80.

[4] - A blow up result for the critical exponents in cones, Israel J. Math. 67 (1989), 129-136.

[5] M.H. Protter and H.F. Weinberger, "Maximum principles in Differential Equations," Prentice-Hall, 1967.

[6] G.N. Watson, "A treatise on the Theory of Bessel Functions," Cambridge University Press, London/New York, 1944, pp. 395.

University of Tsukuba

Institute of Mathematics

Ibaraki, 305

Japan 